\title{
Observer analysis and synthesis for perturbed Lipschitz systems under noisy time-varying measurements *
}

\author{
Etienne Lucien $^{a}$, Hetel Laurentiu ${ }^{\mathrm{b}}$, Efimov Denis ${ }^{\mathrm{c}}$ \\ ${ }^{a}$ DIA, Institut Mine Télécom Lille Douai, 764 Boulevard Lahure, 59500 Douai, France \\ ${ }^{\mathrm{b}}$ CRIStAL, CNRS UMR 9189, Centrale Lille, 59650 Villeneuve d'Ascq, France \\ ${ }^{\mathrm{c}}$ Inria, Univ. Lille, CNRS, UMR 9189 - CRIStAL, 59000 Lille, France
}

\begin{abstract}
In this paper the observer synthesis problem is studied for nonlinear Lipschitz systems with noisy time-varying sampling and bounded state perturbations. To establish criteria for robust convergence of the observer, we model the impact of sampling by a reset integrator operator. First, generic conditions for the input-to-state stability of a sampled-data system are presented. Second, it is shown how to derive a tractable numerical criterion for the synthesis of a sampled-data Luenberger observer. Then, new conditions for robustness analysis of a known observation gain are given.
\end{abstract}

Key words: Observer, Sampled-data systems, Aperiodic Sampling, Lipschitz Nonlinear systems, Robustness, LMI

\section{Introduction}

Nowadays, in a large variety of control systems sensing is done discretely in time and information is sent through communication networks to a computer for state estimation. Aperiodic communications are an intrinsic characteristic of a networked environment $[9,18,10]$. Therefore, there is an increasing interest in the development of estimation methods which take into account aperiodic sampling. Here, we will deal with the estimation problem for a perturbed Lipschitz nonlinear system. In the unperturbed case, the problem has been addressed using various approaches. In [4] and [13] a high-gain approach is considered relying on the computation of normal forms. The works [5, 11] use the approximation of reachable sets based on convex embeddings. Impulsive models and time-varying Lyapunov functions have been used in $[6,3,16]$. A classical issue when considering observer design is the problem of "robustness", i.e. how to characterize the degradation of the estimation quality when noise and perturbation affect, respectively, the output and the plant. Despite the high relevance of this problem, there is a lack of robust analysis methods for aperiodically sampled observation schemes.

In this article, for the case of Lipschitz nonlinear systems, we will study the Input-to-State Stability (ISS) properties of a classical Luenberger observer subject to an aperiodically sampled implementation. Unlike the existing approaches, relaying on the computation of normal forms, convex embeddings or time-varying Lyapunov functions for impulsive systems, here we propose a radically different method, based on an input/output interconnection approach. The effect of sampling in the estimation loop is captured by a reset integrator operator. The observer design problem is studied by exploiting conditions inspired by the Dissipativity Theory [2]. The approach allows to analyse the effect of perturbation (in terms of ISS) on the observation error dynamics.

\footnotetext{
‡ This work has been partially supported by the ANR project DIGITSLID

Email addresses: lucien.etienne@imt-lille-douai.fr (Etienne Lucien), laurentiu.hetel@centralelille.fr (Hetel Laurentiu), denis.efimov@inria.fr (Efimov Denis).
} 
The remainder of this paper is organized as follows. Section 2 is dedicated to the problem statement. In Section 3 , preliminaries on the reset integrator are provided. In Section 4, generic conditions for robust observer analysis are given. In Section 5, numerically tractable conditions for observer design and robustness analysis are provided in terms of LMI's. At last, in Section 6, we illustrate our approach by numerical simulations.

Notation: For a square symmetric matrix $P, \lambda_{\max }(P)\left(\lambda_{\min }(P)\right)$ denotes the largest (smallest) eigenvalue; $P>0$, $(P<0)$ means that $P$ is positive definite (negative definite). In a symmetric matrix, the symbol $\star$ denotes the elements that can be induced by symmetry. For a matrix $A, H e(A):=A+A^{\prime}$. $\mathbb{R}_{\geq 0}$ corresponds to the set of non-negative real numbers. Given $r \in \mathbb{N}$, the set $\Delta_{r}$ denotes the unit simplex, $\Delta_{r}:=\left\{\lambda \in \mathbb{R}_{\geq 0}^{r}: \sum_{i=1}^{r} \lambda_{i}=1\right\}$. The space of functions $f:\left[t_{0}, t_{1}\right) \rightarrow \mathbb{R}^{m}$ which are quadratically integrable over the interval $\left[t_{0}, t_{1}\right)$ is denoted as $L_{2}\left[t_{0}, t_{1}\right)$ while $L_{2 e}$ denotes the space of functions which are quadratically integrable over every bounded interval of $\mathbb{R}_{\geq 0}$. For a bounded function $f: \mathbb{R}_{\geq 0} \rightarrow \mathbb{R}^{m}$, we write $|f|_{\infty}=\sup _{t \in \mathbb{R}_{\geq 0}}|f(t)|$.

\section{Problem statement}

We consider a nonlinear Lipschitz system of the form

$$
\begin{aligned}
\dot{x}(t) & =A x(t)+B u(t)+\mathcal{G} \phi(\mathcal{H} x(t))+\delta(t), t \geq 0, \\
\nu_{k} & =C x\left(t_{k}\right)+\sigma\left(t_{k}\right), k \in \mathbb{N},
\end{aligned}
$$

where $x \in \mathbb{R}^{n}$ is the state with initial condition $x(0)=x_{0}$ and $u \in \mathbb{R}^{l}$ is the input applied to the system. Here $A \in \mathbb{R}^{n \times n}, B \in \mathbb{R}^{n \times l}, C \in \mathbb{R}^{q \times n}, \mathcal{G} \in \mathbb{R}^{n \times m}, \mathcal{H} \in \mathbb{R}^{m \times n}$ and $\phi: \mathbb{R}^{m} \rightarrow \mathbb{R}^{m}$. The nonlinear term $\phi$ satisfies the Lipschitz condition: $|\phi(a)-\phi(b)| \leq K_{\phi}|a-b|$, for all $(a, b) \in \mathbb{R}^{m} \times \mathbb{R}^{m}$, for some $K_{\phi}>0$. It is assumed that $u($.$) is piecewise continuous and bounded. \left(t_{k}\right)_{k \in \mathbb{N}}$ denotes the sequence of sampling times, We assume that $t_{0}=0<t_{k+1}-t_{k} \leq \bar{h}$ and $\lim _{k \rightarrow \infty} t_{k}=\infty$. Here $\bar{h}$ denotes the maximum inter-sampling time. $\nu_{k} \in \mathbb{R}^{q}$ is the noisy sampled output available at time $t_{k}$ with the noise $\sigma\left(t_{k}\right) \in \mathbb{R}^{q}$, and $\delta(t) \in \mathbb{R}^{n}$ is a perturbation. It is furthermore assumed that $|\delta|_{\infty}<+\infty$ and $|\sigma|_{\infty}<+\infty$. We consider the following sampled-data implementation of the Luenberger observer:

$$
\begin{aligned}
\dot{\hat{x}}(t)= & A \hat{x}(t)+B u+\mathcal{G} \phi(\mathcal{H} \hat{x}(t)) \\
& +L\left(C \hat{x}\left(t_{k}\right)-\nu_{k}\right), \forall t \in\left[t_{k}, t_{k+1}\right),
\end{aligned}
$$

where $\hat{x} \in \mathbb{R}^{n}$ is the state of the observer, and $L \in \mathbb{R}^{n \times q}$ is the observer gain. The goal is to design an observer gain $L$ which takes into account the effect of sampling and to quantify the robustness of a known observer with respect to the perturbation $|\delta|$ and $|\sigma|$.

\section{Preliminaries}

Consider the observation error $z=x-\hat{x}$. Since the observer (2) uses sampled measurements $x\left(t_{k}\right)$ and $\hat{x}\left(t_{k}\right)$, the error dynamics also depends on the perturbation induced by the sampling $w(t)=z(t)-z\left(t_{k}\right)$. Using (1), (2), the error dynamics in the sampled case is given by

$$
\begin{aligned}
\dot{z}(t)= & (A+L C) z(t)+\Psi(x(t), z(t)) \\
& +L C w(t)+L \sigma\left(t_{k}\right)+\delta(t), \forall t \in\left[t_{k}, t_{k+1}\right),
\end{aligned}
$$

where $\Psi(x, z)=\mathcal{G}(\phi(\mathcal{H} x)-\phi(\mathcal{H} x-\mathcal{H} z))$. The idea of the input/output interconnection approach is to characterize the sampling induced perturbation $w$ by an operator which has as argument the derivative of the observation error $\dot{z}$. Note that $\forall t \in\left[t_{k}, t_{k+1}\right)$

$$
w(t)=z\left(t_{k}\right)-z(t)=-\int_{t_{k}}^{t} \dot{z}(s) d s .
$$

Defining $y=\dot{z}$, the sampling induced perturbation can be represented as $w=\Omega y$, where the operator $\Omega: L_{2 e} \rightarrow L_{2 e}$ is the following reset integrator :

$$
\Omega:\left\{\begin{array}{l}
\dot{\eta}=y, \quad \forall t \in\left[t_{k}, t_{k+1}\right) \\
\eta\left(t_{k}\right)=0, \quad \forall k \in \mathbb{N} \\
w(t)=-\eta(t), \quad t \geq 0
\end{array}\right.
$$


The operator $\Omega$ has been studied in the literature and has several properties that can be useful for the analysis of sampled-data systems (see the work of [8] and [12] where it was used for deriving stability conditions for LTI sampled-data systems using a frequency domain approach). For example, $\Omega$ has a bounded $L_{2}$ gain over the intervals $\left[t_{k}, t_{k+1}\right)$, i.e.

$$
\int_{t_{k}}^{t}|\Omega y(\theta)|^{2} d \theta \leq \frac{4 \bar{h}^{2}}{\pi^{2}} \int_{t_{k}}^{t}|y(\theta)|^{2} d \theta
$$

for all $t \in\left[t_{k}, t_{k+1}\right)$. This is a direct consequence of Wirtinger's inequality [7]. In a more general setting [15, 14], the properties of the reset integrator $\Omega$ were described using inequalities of the form

$$
\int_{t_{k}}^{t} s(y(\theta), \Omega(y(\theta))) d \theta \leq 0, \forall t \in\left[t_{k}, t_{k+1}\right),
$$

where $s: \mathbb{R}^{n} \times \mathbb{R}^{n} \rightarrow \mathbb{R}$ is a continuous function. For example, the bounded $L_{2}$ gain condition (6) can be expressed in the form (7) with $s(y, w)=|w|^{2}-\frac{4 \bar{h}^{2}}{\pi^{2}}|y|^{2}$.

In the following sections we will see how conditions of the form (7) can be used to design observers and to establish ISS criteria for the observer error dynamics (3).

\section{Generic conditions for convergence}

Consider a more general class of nonlinear systems

$$
\left\{\begin{array}{l}
\dot{z}=f(z, w, \Gamma, v), z(0) \in \mathbb{R}^{n}, \\
w(t)=z\left(t_{k}\right)-z(t), \forall t \in\left[t_{k}, t_{k+1}\right), k \in \mathbb{N},
\end{array}\right.
$$

where $f: \mathbb{R}^{n} \times \mathbb{R}^{n} \times \mathbb{R}^{n} \times \mathcal{V} \rightarrow \mathbb{R}^{n}$ is Lipschitz continuous in all arguments, $w$ is the sampling induced error, $\Gamma$ is a bounded perturbation with $|\Gamma|_{\infty}=\bar{\Gamma}$ and $v: \mathbb{R}_{\geq 0} \rightarrow \mathcal{V}$ is a continuous function defined over a closed set $\mathcal{V}$ that may account for uncertainties or exogenous inputs. System (3) can be rewritten in the form of (8) with $f(z, w, \Gamma, v)=(A+L C) z+L C w+\Psi(v, z)+\Gamma$, where $\Gamma=L \sigma+\delta$. Before presenting a generic result concerning the ISS properties of system (8) we present the main assumptions used for establishing our results.

H.1. There exists a continuous $s: \mathbb{R}^{n} \times \mathbb{R}^{n} \rightarrow \mathbb{R}$ which satisfies the following property

$$
\int_{t_{k}}^{t} s(\dot{z}(\theta), w(\theta)) d \theta \leq 0, \forall t \in\left[t_{k}, t_{k+1}\right),
$$

along the trajectories of $(8)$.

As seen in Section 3, typical examples of functions $s$ satisfying H.1 can be found by characterizing the input-output properties of the reset integrator $\Omega$ described in (5) using constraints of the form (7).

H.2. There exist a continuously differentiable function $V: \mathbb{R}^{n} \rightarrow \mathbb{R}_{\geq 0}$, and $c_{1}, c_{2}, \alpha>0, p \geq 1, \gamma \geq 0$ with $c_{1}|z|^{p} \leq V(z) \leq c_{2}|z|^{p}, \forall z \in \mathbb{R}^{n}$, such that

$$
\dot{V}(z(t))+\alpha V(z(t)) \leq e^{-\alpha\left(t-t_{k}\right)} s(\dot{z}(t), w(t))+\gamma^{p}|\Gamma(t)|^{p}
$$

for all $t \in\left[t_{k}, t_{k+1}\right)$, along the trajectories $z($.$) of (8).$

The function $V$ satisfying H.2 can be seen as an ISS Lyapunov function for system (8) with respect to $\Gamma$. At the same time, the function acts as a kind of storage function which ensures the (exponential) dissipativity of system (8) with respect to the supply function $s(\dot{z}, w)$ over a sampling interval. The following theorem shows that H.1 and H.2 are sufficient for the ISS of system (8).

Theorem 1 Consider system (8) and Assumptions H.1-H.2. Then for any $z(0) \in \mathbb{R}^{n}$ and $\bar{\Gamma} \geq 0$ the solutions of system (8) satisfy $|z(t)| \leq \sqrt[p]{\frac{c_{2}}{c_{1}}} e^{-\frac{\alpha t}{p}}|z(0)|+\frac{\gamma \bar{\Gamma}}{\sqrt[p]{c_{1} \alpha}}$, for all $t \geq 0$, i.e. system (8) is ISS. 
Proof 1 Using H.2 and noting that $\gamma^{p}|\Gamma|^{p} \leq \gamma^{p} \bar{\Gamma}^{p}$, on the time interval $\left[t_{k}, t_{k+1}\right)$ we have

$$
\begin{aligned}
& e^{\alpha\left(t-t_{k}\right)}(\dot{V}(z(t))+\alpha V(z(t))) \\
& \quad \leq s(\dot{z}(t), w(t))+e^{\alpha\left(t-t_{k}\right)} \gamma^{p} \bar{\Gamma}^{p} .
\end{aligned}
$$

Integrating from $t_{k}$ to some $t$ in $\left[t_{k}, t_{k+1}\right)$, yields

$$
\begin{aligned}
e^{\alpha\left(t-t_{k}\right)} V(z(t))-V\left(z\left(t_{k}\right)\right) & \\
& \leq \int_{t_{k}}^{t} s(\dot{z}(\theta), w(\theta))+e^{\alpha\left(\theta-t_{k}\right)} \gamma^{p} \bar{\Gamma}^{p} d \theta
\end{aligned}
$$

Therefore, in virtue of $H .1$, one has $\forall t \in\left[t_{k}, t_{k+1}\right)$

$V(z(t)) \leq e^{-\alpha\left(t-t_{k}\right)} V\left(z\left(t_{k}\right)\right)+\frac{\gamma^{p} \bar{\Gamma}^{p}}{\alpha}\left[1-e^{-\alpha\left(t-t_{k}\right)}\right]$

By iteration, $V(z(t)) \leq V(z(0)) e^{-\alpha t}+\frac{\gamma^{p} \bar{\Gamma}^{p}}{\alpha}\left(1-e^{-\alpha t}\right)$ for all $t>0$. This leads to $c_{1}|z(t)|^{p} \leq V(z(t)) \leq c_{2}|z(0)|^{p} e^{-\alpha t}+$ $\gamma^{p} \bar{\Gamma}^{p} \alpha^{-1}$, for all $t>0$.

Let us now reconsider the dynamics of the observation error (3) and a supply function $s$ characterizing the reset integrator (5) by a condition of the form (7). As a direct application of Theorem 1 to the case of system (3), investigating the ISS of (3) leads to the following problem:

Problem 1. Find $\alpha, c_{1}, c_{2}>0, p \geq 1, \gamma \geq 0$ and a continuously differentiable function $V: \mathbb{R}^{n} \rightarrow \mathbb{R}_{\geq 0}$, with $c_{1}|z|^{p} \leq V(z) \leq c_{2}|z|^{p}, \forall z \in \mathbb{R}^{n}$, such that the following set of Differential Inequalities is satisfied

$$
\left\{\begin{array}{l}
\frac{\partial V}{\partial z} y+\alpha V(z) \leq e^{-\alpha \tau} s(y, w)+\gamma^{p}|\Gamma|^{p} \\
\forall \tau \in[0, \bar{h}], x, y, z, w, \Gamma \in \mathbb{R}^{n} \text { s.t. } \\
y=(A+L C) z+L C w+\Psi(x, z)+\Gamma
\end{array}\right.
$$

The set of inequalities (9) provide generic conditions for the ISS of the observation error (3). However, the conditions are not constructive. In the following section, we show how tractable ISS criteria can obtained.

\section{Tractable conditions}

In order to transform Problem 1 into a numerically tractable one, the structure of the nonlinear function $\Psi(x, z)=$ $\mathcal{G}(\phi(\mathcal{H} x)-\phi(\mathcal{H} x-\mathcal{H} z))$ can be used. Since $\phi$ is Lipschitz, it is possible to find a finite set of $p$ matrices $\Psi_{i} \in$ $\mathbb{R}^{n \times n}, i \in \mathcal{R}=\{1,2, \ldots, r\}$, such that $\Psi(x, z) \in \operatorname{Conv}\left\{\Psi_{i} z\right\}_{i \in \mathcal{R}}$ for all $x, z \in \mathbb{R}^{n}$ - see for instance [17] where a constructive procedure is given.

Problem 2. Find $\alpha, c_{1}, c_{2}>0, p \geq 1, \gamma \geq 0$ and a continuously differentiable function $V: \mathbb{R}^{n} \rightarrow \mathbb{R}_{\geq 0}$, with $c_{1}|z|^{p} \leq V(z) \leq c_{2}|z|^{p}, \forall z \in \mathbb{R}^{n}$, such that the following set of Differential Inequalities is satisfied

$$
\left\{\begin{array}{l}
\frac{\partial V}{\partial z} y+\alpha V(z) \leq e^{-\alpha \tau} s(y, w)+\gamma^{p}|\Gamma|^{p} \\
\forall \tau \in[0, \bar{h}], v \in \Delta_{r}, y, z, w, \Gamma \in \mathbb{R}^{n} \text { s.t. } \\
y=(R(v)+L C) z+L C w+\Gamma
\end{array}\right.
$$

where $R(v)=A+\sum_{i \in \mathcal{R}} v_{i} \Psi_{i}, v \in \Delta_{r}$ 
Remark 1 If $\left(V, \alpha, c_{1}, c_{2}, p, \gamma\right)$ is a solution to Problem 2 then it is also a solution to Problem 1:

Indeed, take any $(\tau, x, y, z, \Gamma) \in[0, \bar{h}] \times \mathbb{R}^{4 n}$. By definition of $\Psi_{i}$ there exist $v \in \Delta_{r}$ such that $R(v) z=A z+\Psi(x, z)$. Since $y=(R(v)+L C) z+L C w+\Gamma \Rightarrow \frac{\partial V}{\partial z} y+\alpha V(z) \leq e^{-\alpha \tau} s(y, w)+\gamma^{p}|\Gamma|^{p}$, we have that $y=(A+L C) z+L C w+$ $\Psi(x, z)+\Gamma \Rightarrow \frac{\partial V}{\partial z} y+\alpha V(z) \leq e^{-\alpha \tau} s(y, w)+\gamma^{p}|\Gamma|^{p}$.

Remark 2 The advantage of the formulation (10) consist in the fact that, for simple choices of functions $V$ and $s$, tractable criteria in terms of matrix inequities can be derived. An alternative formulation can be obtained by directly using the Lipschitz constant of $\phi$, i.e. $|\phi(\mathcal{H} x)-\phi(\mathcal{H} x-\mathcal{H} z)| \leq K_{\phi}|\mathcal{H} z|$, for all $(x, z) \in \mathbb{R}^{n} \times \mathbb{R}^{n}$, instead of the polytopic embedding $\Psi(x, z) \in \operatorname{Conv}\left\{\Psi_{i} z\right\}_{i \in \mathcal{R}}$ for all $x, z \in \mathbb{R}^{n}$. However, it might lead to more conservative stability criteria [17].

\subsection{Perturbation free case}

Consider the perturbation free case $(\bar{\Gamma}=0)$ and functions $V(z)=z^{\prime} P z$ and $s(y, w)=\left(y^{\prime} w^{\prime}\right) \Pi\left(y^{\prime} w^{\prime}\right)^{\prime}$ with $P=$ $P^{\prime}>0, P \in \mathbb{R}^{n \times n}, \Pi=\Pi^{\prime} \in \mathbb{R}^{2 n \times 2 n}$. Setting $\xi^{\prime}=\left(z^{\prime} y^{\prime} w^{\prime}\right)$, condition (10) leads to

$$
\left\{\begin{array}{l}
\xi^{\prime} \mathcal{M}(P, \Pi, \tau) \xi \leq 0 \\
\forall \xi \in \mathbb{R}^{3 n}, \tau \in[0, \bar{h}], v \in \Delta_{r} \text { s.t. } \\
H(v) \xi=0
\end{array}\right.
$$

where

$$
\mathcal{M}(P, \Pi, \tau)=\left[\begin{array}{c|cc}
\alpha P & P & 0 \\
\hline \star & -e^{-\alpha \tau} \Pi \\
\star & &
\end{array}\right]
$$

and $H(v)=[R(v)+L C,-I, L C]$. Using Finsler's Lemma [1], for a prescribed decay rate $\alpha>0$, a sufficient set of LMIs is obtained:

LMI Problem: $\exists P \in \mathbb{R}^{n \times n}, \bar{G} \in \mathbb{R}^{n \times 3 n}$ such that

$$
P>0, \mathcal{M}(P, \Pi, \tau)+\bar{G}^{\prime} H(v)+H(v)^{\prime} \bar{G}<0,
$$

for all $(\tau, v) \in[0, \bar{h}] \times \Delta_{r}$.

The introduction of the matrix $\bar{G}$ introduces some conservatism in the formulation (13) w.r.t. the condition (11). However, it can be easily transformed into a finite set of LMIs using standard convexity arguments. This leads to a simple numerical test for the existence of solutions to Problem 1 (in the perturbation free case). In addition the procedure can be used for observer design. The results are reported below.

Proposition 1 Consider system (1) with $|\delta|_{\infty}=|\sigma|_{\infty}=0$, the observer (2), the error dynamics (3) and the reset integrator (5). Let there exist a function

$$
s(y, w)=\left(y^{\prime} w^{\prime}\right) \Pi\left(y^{\prime} w^{\prime}\right)^{\prime}=y^{\prime} \Pi_{1} y+2 y^{\prime} \Pi_{2} w+w^{\prime} \Pi_{3} w
$$

satisfying condition (7) and a finite set of matrices $\Psi_{i} \in \mathbb{R}^{n \times n}, i \in \mathcal{R}=\{1,2, \ldots, r\}$, such that $\Psi(x, z) \in$ Conv $\left\{\Psi_{i} z\right\}_{i \in \mathcal{R}}$ for all $x, z \in \mathbb{R}^{n}$. Given $\alpha>0$, assume that there exist matrices $P=P^{\prime}>0, G \in \mathbb{R}^{n \times n}, Q \in \mathbb{R}^{n \times q}$ such that the following set of LMIs is feasible:

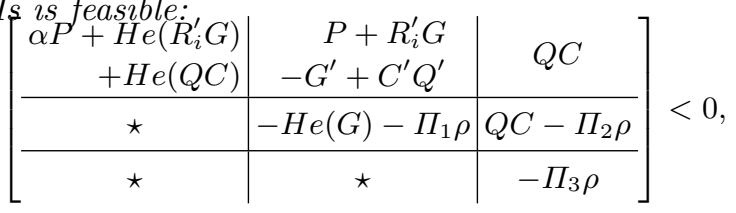

$\forall(i, \rho) \in \mathcal{R} \times\left\{1, e^{-\alpha \bar{h}}\right\}$, where the notation $R_{i}=A+\Psi_{i}$ has been used. Then system (3) with the observation gain $L=\left(G^{\prime}\right)^{-1} Q$, is exponentially stable. 
Proof 2 Assume (14) holds. Using the fact that $H(v) \in \operatorname{Conv}\left\{H_{i}\right\}_{i \in \mathcal{R}}$, where $H_{i}=\left[R_{i}+L C,-I, L C\right]$, and simple convexity arguments, leads to

$\left[\begin{array}{r|c|r}\alpha P+H e\left(R^{\prime}(v) G\right) & P+R^{\prime}(v) G & Q \\ +H e(Q C) & -G^{\prime}+C^{\prime} Q^{\prime} & \\ \hline \star & -H e(G)-\Pi_{1} e^{-\alpha \tau} & -\Pi_{2} e^{-\alpha \tau} \\ +Q C \\ \hline \star & \star & -\Pi_{3} e^{-\alpha \tau}\end{array}\right]<0$,

for all $(\tau, v) \in[0, \bar{h}] \times \Delta_{r}$. The latter is the same as (13) with $\bar{G}=[G, G, 0]$ and $L=\left(G^{\prime}\right)^{-1} Q$. Therefore $\left(V, \alpha, \lambda_{\min }(P), \lambda_{\max }(P), 2,0\right)$ is a solution to Problem 1. Since condition (7) holds by assumption, there exists a quadratic function $V(z)=z^{\prime} P z$ satisfying Assumptions H.1, H.2 of Theorem 1.

Proposition 1 provides numerically tractable conditions for the design of a sampled-data observer. Due to the fact that several approximations are used in our methodology, the existence of matrices $P, G$ and $Q$ satisfying the set of LMIs (14), is a sufficient only design condition.

\subsection{Perturbation analysis}

The methodology used in Section 5.1 can be applied for analysing of the effect of perturbations.

Proposition 2 Consider system (1) with $\Gamma=L \sigma+\delta$, the observer (2), the error dynamics (3) and the reset integrator (5). Let there exist a quadratic function

$$
s(y, w)=\left(y^{\prime} w^{\prime}\right) \Pi\left(y^{\prime} w^{\prime}\right)^{\prime}=y^{\prime} \Pi_{1} y+2 y^{\prime} \Pi_{2} w+w^{\prime} \Pi_{3} w
$$

satisfying condition (7) and a finite set of matrices $\Psi_{i} \in \mathbb{R}^{n \times n}, i \in \mathcal{R}=\{1,2, \ldots, r\}$, such that $\Psi(x, z) \in$ Conv $\left\{\Psi_{i} z\right\}_{i \in \mathcal{R}}$ for all $x, z \in \mathbb{R}^{n}$. Given $\alpha>0$, assume that there exist $\gamma \geq 0$ and matrices $P=P^{\prime}>0, G_{i} \in$ $\mathbb{R}^{n \times n}, i=1, \ldots, 4$, such that the following set of $L M, I$ s is feasible:

\begin{tabular}{|c|c|c|c|}
\hline $\begin{array}{l}\text { lowing, set } \\
H e\left(G_{1}^{\prime} R_{i}\right)\end{array}$ & of $\begin{array}{c}L M, I S \\
R_{i} G_{2} \\
D\end{array}$ & $\begin{array}{r}\text { s feasible: } \\
R_{i} G_{3}\end{array}$ & $\tilde{R}_{i}^{\prime} G_{4}$ \\
\hline & $\begin{array}{l}+P-G_{1}^{\prime} \\
-\mathrm{He}\left(G_{2}\right)\end{array}$ & $\begin{array}{r}+G_{1}^{\prime} L C \\
G_{2}^{\prime} L C\end{array}$ & $\frac{+G_{1}^{\prime}}{G_{2}^{\prime}}$ \\
\hline 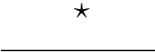 & $-\Pi_{1} \rho$ & $-G_{3}-\Pi_{2} \rho$ & $-G_{4}$ \\
\hline ๙ & ๙ & $\begin{array}{r}H e\left(G_{3}^{\prime} L C\right) \\
-\Pi_{3} \rho\end{array}$ & $\begin{array}{r}G_{3}^{\prime}+ \\
C^{\prime} L^{\prime} G_{4}\end{array}$ \\
\hline ^ & 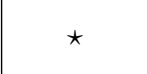 & $\star$ & $\begin{array}{r}\mathrm{He}\left(G_{4}\right) \\
\quad-\gamma^{2} I\end{array}$ \\
\hline
\end{tabular}

$\forall(i, \rho) \in \mathcal{R} \times\left\{1, e^{-\alpha \bar{h}}\right\}$, where the notation $\tilde{R}_{i}=A+\Psi_{i}+$ LC has been used. Then system (3) is ISS i.e. $|z(t)| \leq$ $\sqrt{\frac{\lambda_{\max }(P)}{\lambda_{\min }(P)}} e^{-\frac{\alpha t}{2}}|z(0)|+\frac{\gamma|\Gamma(t)|_{\infty}}{\sqrt{\lambda_{\min }(P) \alpha}}, \forall t \geq 0$.

Proof 3 When $\bar{\Gamma} \neq 0$, for quadratic functions $V(z)=z^{\prime} P z$ and $s(y, w)=\left(y^{\prime} w^{\prime}\right) \Pi\left(y^{\prime} w^{\prime}\right)^{\prime}$, condition (10) can be expressed in the form (11) with $\xi=\left[z^{\prime}, y^{\prime}, w^{\prime}, \Gamma^{\prime}\right]^{\prime}$ and

$$
\mathcal{M}(P, \Pi, \tau)=\left[\begin{array}{c|cc|c}
\alpha P & P & 0 & 0 \\
\hline \star & -e^{-\alpha \tau} \Pi & 0 \\
\star & & & 0 \\
\hline \star & \star & \star & \gamma^{2} I
\end{array}\right] .
$$

Following the same steps as in Section 5.1, the ISS - analyzis leads to an LMI problem of the form (13) with $\mathcal{M}(P, \Pi, \tau)$ given by (16), $H(v)=[R(v)+L C,-I, L C, I]$ and $\bar{G} \in \mathbb{R}^{n \times 4 n}$. Using $\bar{G}=\left[G_{1}, G_{2}, G_{3}, G_{4}\right]$ and the fact that $H(v) \in \operatorname{Conv}\left\{H_{i}\right\}_{i \in \mathcal{R}}$, where $H_{i}=\left[R_{i}+L C,-I, L C, I\right]$, and simple convexity arguments leads to the set of LMIs (15). The latter is sufficient for (13) to hold. Therefore, $\left(V, \alpha, \lambda_{\min }(P), \lambda_{\max }(P), 2, \gamma\right)$ is a solution to Problem 1. Since condition (7) holds, there exists a quadratic function $V(z)=z^{\prime} P z$ satisfying the assumptions of Theorem 1. 


\section{Example: DC drive}

Consider the example of a single-link direct-drive manipulator actuated by a permanent magnet DC brush motor $[11]$

$$
\dot{x}=\left(\begin{array}{c}
x_{2} \\
-2 \sin \left(x_{1}\right)-3 x_{2}+x_{3} \\
u-x_{2}-x_{3}
\end{array}\right),
$$

$\nu_{k}=\left(x_{1}\left(t_{k}\right)+0.2 \sin \left(1000 t_{k}\right), 0,0\right)$. For $\alpha=1, \delta(t)=0, \bar{h}=0.4$, considering the specific $\Pi=\left[\begin{array}{c}X \\ \star\end{array}-\left(\frac{2 h}{\pi}\right)^{2} X\right]$ from [14] and using Proposition 1 with $X>0$ and $Y>0$ as additional LMI variables, one finds the observer gain $L=[-2.2,0.16,0.45]^{\prime}$. Applying Proposition 2, one finds that the system is ISS with parameters $\gamma=2.7, c_{1}=1$. The results are illustrated in Fig. 1, where the observer scheme allows for robust observation.

a)

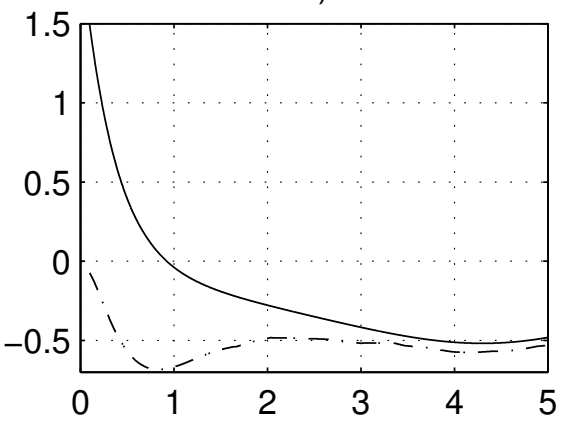

b)

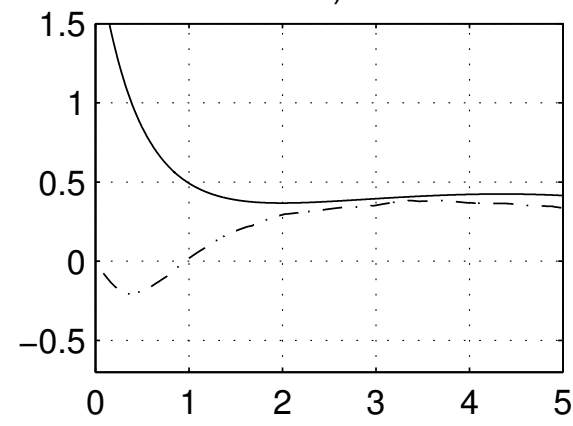

c)

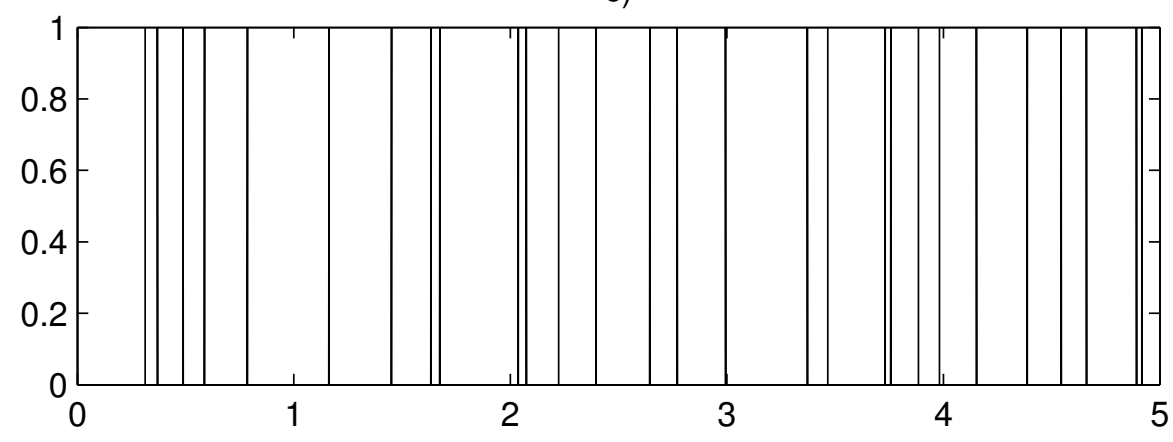

Fig. 1. a) unmeasured state $x_{2}$ (solid line) and estimation $\hat{x}_{2}$ (dashed line) b) unmeasured state $x_{3}$ (solid line) and estimation $\hat{x}_{3}$ (dashed line). c) Sampling instants indicated by vertical lines.

\section{Conclusion}

In this work the problems of observer synthesis and robustness analysis of an observer for a nonlinear Lipschitz system with sampled measurements have been investigated. The case where the state is perturbed and where noisy measurements are sampled aperiodically has been studied. Our analysis is based on the use of a reset integrator operator, which captures the effect of the error induced by sampling. Both theoretical and numerically tractable conditions were provided. An LMI criterion for the synthesis of a sampled-data Luenberger observer was given. Criteria for robustness analysis (in terms of ISS) have been derived. In the future we intend to study the design of more advanced sampled-data observer structures, which may handle more effectively the impact of perturbations. 


\section{References}

[1] S. Boyd, L. El-Ghaoui, E. Feron, V. Balakrishnan, and E. Yaz. Linear matrix inequalities in system and control theory. Proceedings of the IEEE, 85(4):698-699, 1997.

[2] B. Brogliato, R. Lozano, B. Maschke, and O. Egeland. Dissipative systems analysis and control. Theory and Applications, 2nd ed. London: Springer-Verlag, 2007.

[3] W.-H. Chen, D.-X. Li, and X. Lu. Impulsive observers with variable update intervals for lipschitz nonlinear time-delay systems. International Journal of Systems Science, 44(10):1934-1947, 2013.

[4] F. Deza, E. Busvelle, J. Gauthier, and D. Rakotopara. High gain estimation for nonlinear systems. Systems 86 control letters, 18(4):295-299, 1992.

[5] T. N. Dinh, V. Andrieu, M. Nadri, and U. Serres. Continuous-discrete time observer design for lipschitz systems with sampled measurements. IEEE Transactions on Automatic Control, 60(3):787-792, 2015.

[6] L. Etienne, L. Hetel, D. Efimov, and M. Petreczky. Observer synthesis under time-varying sampling for lipschitz nonlinear systems. Automatica, 85:433-440, 2017.

[7] E. Fridman. New Lyapunov - Krasovskii functionals for stability of linear retarded and neutral type systems. Systems \& Control Letters, 43(4):309-319, 2001.

[8] H. Fujioka. Stability analysis of systems with aperiodic sample-and-hold devices. Automatica, 45(3):771-775, 2009.

[9] J. P. Hespanha, P. Naghshtabrizi, and Y. Xu. A survey of recent results in networked control systems. Proceedings of the IEEE, 95(1):138-162, 2007.

[10] L. Hetel, C. Fiter, H. Omran, A. Seuret, E. Fridman, J.-P. Richard, and S. I. Niculescu. Recent developments on the stability of systems with aperiodic sampling: An overview. Automatica, 76:309-335, 2017.

[11] F. Mazenc, V. Andrieu, and M. Malisoff. Design of continuous-discrete observers for time-varying nonlinear systems. Automatica, 57:135-144, 2015.

[12] L. Mirkin. Some remarks on the use of time-varying delay to model sample-and-hold circuits. IEEE Transactions on Automatic Control, 52(6):1109-1112, 2007.

[13] M. Nadri, H. Hammouri, and R. M. Grajales. Observer design for uniformly observable systems with sampled measurements. IEEE Transactions on Automatic Control, 58(3):757-762, 2013.

[14] H. Omran, L. Hetel, M. Petreczky, J.-P. Richard, and F. Lamnabhi-Lagarrigue. Stability analysis of some classes of input-affine nonlinear systems with aperiodic sampled-data control. Automatica, 70:266-274, 2016.

[15] H. Omran, L. Hetel, J.-P. Richard, and F. Lamnabhi-Lagarrigue. Stability analysis of bilinear systems under aperiodic sampled-data control. Automatica, 50(4):1288-1295, 2014.

[16] T. Raff and F. Allgower. Observers with impulsive dynamical behavior for linear and nonlinear continuous-time systems. In Decision and Control, 2007 46th IEEE Conference on, pages 4287-4292. IEEE, 2007.

[17] A. Zemouche, M. Boutayeb, and G. I. Bara. Observers for a class of lipschitz systems with extension to $h_{\infty}$ performance analysis. Systems \& Control Letters, 57(1):18-27, 2008.

[18] W. Zhang, M. S. Branicky, and S. M. Phillips. Stability of networked control systems. IEEE Control Systems, 21(1):84-99, 2001. 